\title{
ACERCAMIENTO A LOS ESTUDIOS REALIZADOS CON MICROFÓSILES EN COSTA RICA
}

\author{
APPROACH TO STUDIES IN COSTA RICA WITH MICROFOSSILS
}

\author{
Julliet Betancur \\ Escuela Centroamericana de Geología, Universidad de Costa Rica. \\ Apdo. 214-2060 UCR, Costa Rica \\ julliet.velez@gmail.com
}

(Recibido: 01/05/2014; aceptado: 10/08/2014)

\begin{abstract}
Costa Rica is carried out from biostratigraphic, paleoecological and paleoclimatic point of view. The most numerous studies are done with foraminifera (planktonic and benthic), which are found in the fossil record since the Jurassic period, with numerous events of extinction and the appearance of new species that makes them a good biostratigraphic tool. Investigations in other minor groups such as radiolarians, testate amoebae, diatoms and pollen, are presented as well.

Keywords: microfossils, foraminifera, radiolarians, thecamoebians, diatoms, pollen

RESUMEN: se realiza una recopilación de los estudios realizados con microfósiles en Costa Rica, con enfoques que varían desde bioestratigráficos, paleoecológicos y paleoclimáticos. Los estudios más numerosos se realizan con los foraminíferos (planctónicos y bentónicos), ya que se encuentran en el registro fósil desde el Jurásico, con numerosos eventos de extinción y aparición de nuevas especies que los hace una buena herramienta bioestratigráfica. También se presenta las investigaciones de otros grupos menores como radiolarios, tecamebas, diatomeas y polen.

Palabras clave: microfósiles, foraminíferos, radiolarios, tecamebas, diatomeas, polen
\end{abstract}




\section{INTRODUCCIÓN}

Se consideran microfósiles, a organismos o a sus restos cuyo estudio requiere el uso de un microscopio, su estudio se denomina micropaleontología. Existe una tendencia a restringir este término al estudio de microfósiles con pared mineral, como foraminíferos, y diferenciarlo de la palinología que estudia los microfósiles de pared orgánica como los granos de polen (Brasier, 1980).

La industria petrolera le ha dado un gran impulso a la micropaleontolgía debido al interés comercial, más que al interés científico. La importancia del entendimiento de los microfósiles ha aumentado con los descubrimientos recientes en rocas Precámbricas; los microfósiles ahora proveen la mayor evidencia de la evolución orgánica a través de más de las tres cuartas partes de la historia de la vida sobre la tierra (Brasier, 1980). Muchos de los microfósiles son el producto de organismos de una sola célula, las eucariotas.

Los eucariotas unicelulares (Protista) aparecieron hace $1500 \mathrm{ma}$, sin embargo, solo son abundantes desde hace 550 ma cuando muchos de ellos adquirieron esqueletos fosilizables (Brasier, 1980).

Los protistas son organismos unicelulares móviles con gran variedad de formas. Los foraminíferos, radiolarios y tecamebas son más afines a los animales, mientras que las diatomeas a las algas. Los restos de plantas se estudian en la paleobotánica, pero las estructuras microscópicas de las algas verdes y rojas (especialmente las calcáreas) y las esporas y polen de plantas terrestres son muy comunes en microfósiles (Brasier, 1980).

Antes de 1940 en Costa Rica, se había realizado investigaciones geológicas principalmente por naturalistas y científicos extranjeros. A partir de este año se inician estudios científicos con carácter geológico-micropaleontológico con los trabajos de Alfonso Segura. Es a partir de 1960 que se inician los estudios formales sobre los foraminíferos fósiles en Costa Rica, principalmente por los trabajos del geólogo costarricense Enrique Malavassi Vargas, quien se especializó en micropaleontología.

\section{MICROFÓSILES EN COSTA RICA}

\section{Foraminíferos}

Los foraminíferos fósiles y actuales se han conocido y estudiado por siglos, fueron mencionados por primera vez por Herodotus en el siglo 5th B.C., quien menciona que las calizas de las pirámides de Egipto contienen macroforaminíferos, nummulites (Boudagher-Fadel, 2008).

Los estudios con microfósiles más abundantes y diversos en Costa Rica se han realizado con foraminíferos planctónicos, que han sido estudiados junto con los foraminíferos bentónicos más que cualquier otro grupo de microfósiles. Los foraminíferos representan el $2.5 \%$ de todas las especies de animales conocidas desde el Cámbrico (Kennett, 1982). Los foraminíferos (del griego: foramen $=$ apertura, ferre: acarrear), aparecen por primera vez en el Jurásico Medio, cerca de 170 ma y se expanden desde el Cretácico Medio en todos los océanos del mundo. Aparecen nuevas especies en el Aptiano (120 ma), el Turoniano (90 $\mathrm{ma}$ ), el Paleoceno (55 ma), y el Mioceno (20 ma), alternando con fases de extinción en el Cenoniano (95 ma), el límite Cretácico/Terciario (60 ma), y en el Eoceno Superior (40 ma). Los foraminíferos planctónicos modernos han evolucionado desde el Terciario Inferior, cuando aparecieron las primeras especies espinosas después del límite Cretáceo/Terciario (Bolli et al., 1985).

Lo anterior los hace una herramienta bioestratigráfica muy útil que ha sido utilizada por Browne (documento que se encuentra en la biblioteca de la Escuela Centroamericana de Geología bajo el nombre: Paleontological and Stratigraphic Report of Limon Province, Costa Rica and Northeastern Panamá, informe interno GRCR-59, 1961), Rivier (1973) y Pizarro (1985), en las localidades de la Formación Uscari, Carbón Dos, Guayacán, ríos Banano, Watsi, Reventazón, Sandbox, quebrada Terciopelo, y las localidades de Carbón Dos y Guayacán.

Azema et al. (1981), realizaron nuevos descubrimientos de afloramientos ricos en foraminíferos planctónicos del Paleoceno, en la costa del Pacífico, que permitió afirmar que la mayor parte, 
o bien, la totalidad del borde occidental de Costa Rica estaba ocupado por el mar en dicha época.

Bolz \& Calvo (2003), presentan el hallazgo de 6 especies de foraminíferos planctónicos del Cretácico Tardío en calizas y tobitas hemipelágicas danianas del Complejo Básico de Quepos, así como, de nuevos afloramientos del basamento del Paleoceno Temprano al norte y noroeste de Quepos, en fila Chonta y Parrita, lo anterior, señala la probable existencia de rocas cretácicas en los niveles inferiores inmediatos del Complejo de Quepos, sugiriendo que las rocas danianas expuestas representan la unidad más joven del basamento.

Las paleoreconstrucciones paleoceanográficas y paleoecológicas se basan en asociaciones de foraminíferos bentónicos (Kennett, 1982), asumiendo que las adaptaciones fisiológicas de las especies no cambian con el tiempo, ni cambia su distribución con la profundidad (Douglas, 1979).

Los estudios paleoecológicos y paleoceanográficos en Costa Rica se han realizado en la Cuenca de Limón (Goudkokk \& Porter, 1942 en: Collins \& Coates, 1999; Cassell, 1986; Cassel \& Sen Gupta, 1989; Betancur, 2014) y en la Formación Venado (Sen Gupta et al., 1986).

Rodríquez (2007), realiza una interpretación paleobatimétrica, biocronológica, y cambios eustáticos con foraminíferos bentónicos para la determinación de la sincronización de la subducción inicial de la dorsal del Coco bajo el Arco de Costa Rica-Panamá y la cantidad de levantamiento asociado.

Los macroforaminíferos son bentónicos y se caracterizan más por su estructura interna, que por su tamaño, y deben ser identificados en sección delgada. Desde 1920, los macroforaminíferos se han utilizado como marcadores bioestratigráficos, al tener la ventaja de ser más abundantes que los moluscos y de que existan biomarcadores desde el Triásico (Boudagher-Fadel, M., 2008).

La primera Lepidocyclina descrita en Costa Rica, fue llamada L. hilli por Cushman, 1920 (en: Mallavassi, 1960), colectada de la caliza de Guayabo. Las otras localidades con macroforaminíferos reportados en las rocas del Cretácico Superior están en la península de Santa Elena
(Baumgartner \& Denyer, 2002; Harrison, 1953); Paleoceno Superior, en punta Guiones (Malavassi, 1962); Eoceno Medio, río Damas (Malavassi, 1960 y 1961) y Formación Parritina (Bolz \& Calvo, 2002); Eoceno Superior, en la caliza de las Ánimas (Lohman \& Brinckman, 1931; Cole, 1953), bahía Junquillal, punta Serrucho, El Cajón, río Parrita. Adicionalmente en la zona de las Ruedas y zona de los Patos, solo determinaciones genéricas (Malavassi, 1960 y 1961); Quebrada Grande con una secuencia desde el Eoceno Superior al Mioceno Inferior (Rivier, 1973); Oligoceno Superior, bajo el puente sobre el río Reventazón (Drooger, 1952) en la carretera de Chitaria a Peralta, punta Pelada (Malavassi, 1960, 1961) y península de Nicoya (Baumgartner et al., 2008).

\section{Radiolarios}

Son marinos, planctónicos y aparecen desde el Cámbrico. La morfología del esqueleto es específica para cada especie y es utilizada en la identificación taxonómica (Brasier, 1980).

A pesar de tener radiolaritas en Costa Rica, solo se reporta un intento de identificación taxonómica en el bloque el Rincón, Burica y Golfito, solo con éxito limitado por la fuerte recristalización y diagénesis que presentaron las muestras (Diserens et al., 2003).

\section{Tecamebas}

Las tecamebas son organismos bentónicos caracterizados por una concha aglutinada o autógena en forma de saco, presente generalmente en depósitos tipo turba, en sedimentos de agua fresca de ríos y lagos y en algunos depósitos de agua salobre. Las tecamebas son muy útiles como indicadores ambientales y paleoambientales, por su gran abundancia y diversidad en especies, amplia distribución, fácil identificación y una buena preservación en los sedimentos. Aunque las conchas fosilizadas han sido encontradas en depósitos tan viejos como 
el Carbonífero, Triásico o Cretácico, la mayoría de los registros provienen del Cuaternario (Loeblich \& Tappan, 1961).

El primer trabajo acerca de tecamebas en Costa Rica, fue realizado en 1920 por el ingeniero agrónomo E. Echeverria, el cuál realizó una expedición a las montañas de Barva a una altitud de 2000 m., y recolectó muestras de musgo (Kufferath, 1929).

Posteriormente Laminger (1973), recolectó muestras de agua en las montañas de la Cordillera de Talamanca (Pacífico y Caribe) y en el cráter del volcán Poas (2400 - 4400 m.s.n.m).

En la laguna del volcán Barva, se recolectaron muestras de sedimento (orillas y fondo del lago), como un estudio de calibración de la variabilidad del ecosistema, diversidad de especies y la respuesta de los organismos a los cambios ecológicos, para interpolarlos luego a estudios en profundidad e inferir cambios paleoambientales y paleoclimáticos.

\section{Diatomeas}

Son algas unicelulares que aparecen en el Jurásico (Brasier, 1980). En Costa Rica se conocen cerca de 33 depósitos de diatomita, ubicados en la vertiente pacífica, de estos 10 son los más importantes y bien diferenciados, todos estrechamente relacionados al vulcanismo ácidointermedio del Terciario tardío-Cuaternario. Los depósitos más grandes, reconocidos hasta hoy, se encuentran en Loma Camastro y Cañas Dulces, ambos en Guanacaste (Mathers et al., 1990).

A pesar de su utilización paleoceanográfica, paleoecológica y bioestratigráfica, en el país, los estudios realizados en diatomitas son escasos, la mayoría de estudios con diatomeas se centran en; ecología actual en lagos y ríos (Michels, 1998a, 1998b; Wydrzycka \& Lange, 2001); paleoecología en el lago de las Morrenas en el Parque Nacional Chirripó con 10.000 años de registro climático (Haberyan \& Horn, 2005), en las diatomitas Loma Camastro (Chávez \& Haberyan, 1996, Gamboa, 2013); y la evaluación del yacimiento para minería en las diatomitas de Quebrada Grande (Gómez, 1992).

\section{Polen}

El primer estudio con polen data de 1964 realizado por Martin en la Cordillera de Talamanca, donde determino un enfriamiento durante el Wisconsiniano (en: Horn, 1985). Los estudios que le siguen se refieren a cambios paleoclimáticos y paleoecologicos, en una gran variedad de escenarios; en un núcleo del proyecto Deep Sea Drilling Project sitio 565, a $42 \mathrm{Km}$ al oeste de la península de Nicoya (Horn, 1985); Cordillera de Talamanca (Hooghiemstra et al., 1992); agricultura prehistórica, fuegos e inundaciones en el Páramo de Chirripó (Horn, 1993), estación biológica La Selva (Kennedy, 1998) y las Cruces (Clement, 1999), lago Cote (Russell, 2001), laguna de Santa Elena (Anchukaitis \& Horn, 2005). En la Formación Río Banano se interpretó la Paleoecología y su relación con el cierre del Itsmo centroamericano (Sandoval, 2009).

\section{CONCLUSIONES}

A pesar de los diversos grupos de microfósiles que se vienen estudiando y utilizando en diferentes disciplinas a nivel mundial, en Costa Rica, los foraminíferos planctónicos y macroforaminíferos son los que revisten la mayor importancia debido a la necesidad de dataciones bioestratigráficas. Aunque se utilizan mayormente las dataciones radiométricas, estás son muy costosas y solo se realizan en instituciones que tienen el presupuesto suficiente para realizarlas fuera del país. Cabe señalar que los estudios paleoclimáticos y paleoecológicos, son herramientas muy útiles para la interpretación paleogeográfica y geológica.

\section{REFERENCIAS BIBLIOGRÁFICAS}

ANCHUKAITIS, K., \& HORN, S., 2005: A 2000-year reconstruction of forest disturbance from southern Pacific Costa Rica.Palaeogeogr. Palaeoclimatol. Palaeoecol. 221: $35-54$. 
AZEMA, J., GLACON, G. \& TOURNON, J., 1981: Nuevos aportes sobre el Paleógeno con foraminíferos planctónicos del margen Pacífico de Costa Rica.- Inst. Geogr. Nac. 26: 57-69.

BAUMGARTNER, C. \& DENYER, P., 2002: Campanian-Maastrichtian limestone with larger foraminifera from Peña Bruja Rock (Santa Elena Península).- Rev. Geol. Amér. Central, 26: 85-89.

BAUMGARTNER, C., BAUMGARTNER, P. \& TSCHUNDIN, P., 2008: Late Oligocene larger foraminifera from Nosara (Nicoya Península, Costa Rica) and indward (Carriacou, Lesser Antilles), calibrated by $87 \mathrm{SR} / 86 \mathrm{Sr}$ isotope stratigraphy.- Rev. Geol. de Amér. Central, 38:33-52.

BETANCUR, J., 2014: Foraminíferos bentónicos como Indicadores paleoecológicos de la Formación Uscari (Mioceno TempranoMedio), cuenca de Limón (sur), Costa Rica.- Rev. Geol. Amér. Central, 50: 193212.

BOLLI, H., SAUNDERS. J.\&PERCH-NIELSEN, K., 1985: Plankton Stratigraphy.-1040 págs. Cambrige University Press, New York.

BOLZ,A.\& CALVO, C., 2002: Calizas Lutetianas del arco interno Paleógeno de Costa Rica.Rev. Geol. Amér. Central, 26: 7-24.

BOLZ, A. \& CALVO, C., 2003: Nuevos datos bioestratigráficos y sedimentológicos sobre el origen del Complejo Básico de Quepos, Costa Rica.- Rev. Geol. Amér. Central, 28: 431-45.

BOUDAGHER-FADEL, M., 2008: Evolution and Geological Significance of Larger Benthic Foraminifera.- En: WIGNALL, P.B. (ed.): Developments in Paleontology \& Stratigraphy, Amsterdan, 21: 1-540.
BRASIER, M. D., 1980: Microfossils.- 193 págs. George Allen \& UNWIN, Londres.

CASSELL, D., 1986: Neogene Foraminifera of the Limon Basin of Costa Rica.- 323 págs. Louisiana State Univ., Louisiana [Tesis Ph.D.].

CASSEL, D.T. \& SEN GUPTA, B.K., 1989: Pliocene foraminifera and environments, Limon Basin of Costa Rica.- J. Paleont. 63(2): 146-157.

CHÁVEZ, L., \& HABERYAN, K. 1996: Diatom assemblages from the Camastro Diatomite de Santa Rosa, Guanacaste, Costa Rica.Rev. Biol. Tropical, 44(2): 899-902

CLEMENT, R.M., 1999: 3000 years of humanvegetation near the Las Cruces Biological Station in southwestern Cost Rica: Paleoecological evidence from laguna Zoncho.- 144 págs. Univ. Tennessee, Knoxville [Tesis M.Sc.].

COLE, W., 1953: Larger foraminifera from the Upper Eocene of Costa Rica.- J. Paleont. 27: 748-749.

COLlinS, L.S., \& COATES, A.G., 1999: A Paleobiotic survey of caribbean faunas from the Caribbean coast of the Isthmus of Panama.- Bull. Amer. Paleont. 357: 351.

DISERENS, M., BAUMGARTNER, P., \& DUMITRICA, P., 2003: Age determination of Late Cretaceous radiolarites in orogenic environmentes: An example from accreted terranes of Southern Costa Rica.- Tenth Meeting of the International Association of Radiolarian Palaeontologists, Lausana, Suiza [Abstracts and Programme]: 49-50.

DOUGLAS, R., G., 1979, Benthic foraminiferal ecology and paleoecology: a review of concepts and methods.- En: LIPPS, J. H. (ed.): Foraminiferal ecology and paleoecology.- Soc. Economic Paleontologists and Mineralogists (SEPM), 6: 21-53. 
DROOGER, C., 1952: Study of American Miogypsinidae.- 80 págs. Univ. Utrecht [Tesis Ph.D.].

GAMBOA, V., 2013: Paleoecología, estratigrafía y sedimentología del depósito de diatomita de Loma Camastro, Liberia-Guanacaste.84 págs. Univ. de Costa Rica, San José [Tesis Lic.].

GÓMEZ, M. 1992: Evaluación de un yacimiento de diatomita en Quebrada Grande-Liberia, Guanacaste (Costa Rica).- 98 págs. Univ. de Costa Rica, San José [Tesis Lic.].

HABERYAN, K., \& HORN, S., 2005: Un registro de diatomeas que cubre 10000 años del lago de las Morrenas 1, Parque Nacional Chirripó, Costa Rica.- En: KAPPELLE, M., \& HORN, S. (ed.): Páramos de Costa Rica.- Inst. Nac. de Biodiversidad (INBio), San José: 275-285.

HARRISON, J., 1953: The Geology of the Santa Elena Peninsula in Costa Rica, Central America.- Proc. $7^{\text {th }}$ Pacific Sci. Congress, Auckland-Christchurch, Nueva Zelanda (2 de febrero al 4 de marzo, 1949), 2:102-114.

HORN, S., 1985: Preliminary pollen analysis of Quaternary sediments from Deep Sea Drilling Project Site 565, Western Costa Rica.- Initial Reports of the Deep Sea Drilling Project, LXXXIV: 533-547.

HORN, S., 1993: Postglacial vegetation and fire history in the Chirripó Páramo of Costa Rica.- Quaternary Res. 40: 107-116.

HOOGHIEMSTRA, H., CLEEG, A., NOLDUS, G., \& KAPPELLE, M., 1992: Upper Quaternary vegetation dynamics and paleoclimatology of the La Chonta bog area (Cordillera de Talamanca, Costa Rica).- J. Quaternary Science, 7(3): 205-225.
KENNEDY, L., 1998: Prehistoric agriculture, fires, and droughts at the La Selva biological station, Costa Rica: Paleoecological evidence from the Cantarrana swamp.- 144 págs. Univ. Tennessee, Knoxville [Tesis M.Sc.].

KENNETT, J.P., 1982: Marine Geology.- 813 págs. Prentice Hall, New Jersey.

KUFFERATH, H., 1929: Algues et protistes muscicoles, corticoles et terrestres récoltés sur la montagne de Barba (Costa Rica).- Annales de Cryptogamie Exotique, 2: 23-52.

LAMINGER, H., 1973: Die Testaceen (Protozoa, Rhizopoda) einiger Hochgebirgsgewässer von Mexiko, Costa Rica und Guatemala.Int. Revue ges. Hydrobiol., 58(2): 273-305.

LOEBLICH, A.R. \& TAPPAN, H., 1961: Suprageneric Classification of the Rhizopodea.- J. Paleontol. 35(2): 245-330.

LOHMAN, W. \& BRINKANN, M., 1931: Uber obereocane Kalke, Gabbros and Andesite von Costa Rica.- Centralblatt für Mineralogie,Geologie und Paläontologie, 10: 553-559.

MALAVASSI, V., 1960: Algunas localidades de Costa Rica con Foraminíferos Grandes.Dirección de Geología, Minas y Petróleo, 3: $1-5$.

MALAVASSI, E., 1961: Some Costa Rican Larger Foraminiferal Localities.- J. Paleont. 35(3): 498-501.

MALAVASSI, E., 1962: Pseudorotalia velascoensis (Cushman), especie índice del Paleoceno Superior en Punta GuionesGuanacaste.- Informes Dirección de Geología, Minas y Petróleo, 11: 1-5. 
MATHERS, S.J., CHÁVEZ, L., ALVARADO, F., \& INGLETHORPE, S., 1990: Detailed Investigations of selected Costa Rica diatomites.- 37 págs. Proyecto AnlgoCostarricense de Minerales Industriales (PACOMI), San José [Inf. Interno $\mathrm{WC} / 90 / 35]$.

MICHELS, A., 1998a: Use of diatoms (Bacillariophyceae) for water quality assessment in two tropical streams in Costa Rica.- Rev. Biol. Trop., 46(6): 143-152.

MICHELS, A., 1998b: Effects of sewage water on diatoms (Bacillariophyceae) and water quality in two tropical streams in Costa Rica.- Rev. Biol. Trop. 46(6): 153175.

PIZARRO, D., 1985: Bioestratigrafía de la Formación Uscari en base a foraminíferos planctónicos/Mioceno Medio a Superior, Costa Rica.- 34 págs. Univ. de Costa Rica, San José [Tesis Lic.].

RIVIER, F., 1973: Contribución estratigráfica sobre la geología de la Cuenca de Limón, zona de Turrialba, Costa Rica.- Publicaciones Geológicas del ICAITI, 4: 149-159.
RODRÍQUEZ, L., 2007: Benthic Foraminiferal Record of the Pleistocene Uplift of the Sedimentary Deposits of the Burica Peninsula (Costa Rica - Panama) as a Result of Cocos Ridge Subduction Beneath the Central American Arc.- 94 págs. Florida Int.Univ., Miami [Tesis M.Sc.].

RUSSELL, M., 2001: Late Holocene environmental history and tephrostratigraphy in Northwestern Costa Rica: A 4000 year record from Lago Cote.- 144 págs. Univ. Tennessee, Knoxville [Tesis M.Sc.].

SANDOVAL, M., 2009: Palinología de la Formación Río Banano (sección Río Carbón Volio, Río Sand Box y Quebrada Veinticinco) Mioceno Superior, Costa Rica.- 56 págs. Univ. de Costa Rica, San José [Tesis Lic.].

SEN GUPTA, B. K., MALAVASSI, L. \& MALAVASSI, E., 1986: Late Miocene shore in Northern Costa Rica: Benthic foraminifera record.- Geology, 14(3): 218-220.

WYDRZYCKA, U. \& LANGE, H., 2001: Las diatomeas (Bacillariophyceae) acidófitas del río Agrio y sitios vinculados con su cuenca, volcán Poás, Costa Rica.Brenesia, 55-56: 1-68. 
